INVESTIGACIÓN

\title{
Caracterización epidemiológica de las personas con discapacidad en Cuba
}

\section{Epidemiological characterization of disabled persons in Cuba}

\author{
Marcia Cobas Ruízi ; Eduardo Zacca Peña ${ }^{\mathrm{II}}$; Francisco Morales Calatayud ${ }^{\mathrm{III}}$; \\ Emelia Icart Pereira ${ }^{\mathrm{IV}}$; Aida Jordán Hernández ${ }^{\mathrm{v}}$; Mitchel Valdés Sosa ${ }^{\mathrm{VI}}$ \\ ILicenciada en Ciencias Sociales. Ministerio de Salud Pública. La Habana, Cuba. \\ ${ }^{\mathrm{II} D o c t o r}$ en Ciencias Médicas. Profesor Titular. Dirección Nacional de Estadística. \\ Ministerio de Salud Pública. La Habana, Cuba. \\ IIIDoctor en Ciencias Médicas. Profesor Titular. Instituto Superior de Ciencias \\ Médicas de La Habana. La Habana, Cuba. \\ ${ }^{\mathrm{IV}}$ Especialista en Pediatría. Centro Médico Psicopedagógico "La Castellana". La \\ Habana, Cuba. \\ `Defectóloga. Centro Médico Psicopedagógico "Benjamín Moreno". La Habana, \\ Cuba. \\ ${ }^{\mathrm{VI}}$ Doctor en Ciencias Médicas. Investigador Titular. Academia de Ciencias de Cuba. \\ La Habana, Cuba.
}

\section{RESUMEN}

Introducción La discapacidad es un tema complejo, de enorme repercusión social y económica y del que se tiene estimados inseguros.

Objetivos Identificar y caracterizar a cada persona con discapacidad en Cuba.

Métodos Investigación epidemiológica descriptiva, de tipo transversal, realizada en el período 2001-2003. El estudio interesó a toda la población cubana y dentro de ella a las personas con discapacidad. La pesquisa activa en la comunidad identificó a las personas registradas y a otras no registradas, con el auxilio de los gobiernos locales y de las organizaciones sociales. Una visita al hogar de la persona con discapacidad permitió aplicar una combinación de entrevistas y exámenes clínicos que facilitaron el proceso de clasificación y registro de estas personas. Se estimó una tasa de cobertura del estudio de $99,9 \%$ del total de personas con discapacidad en el territorio nacional.

Resultados Se estudiaron 366864 personas con discapacidad lo que produjo una tasa de prevalencia de 3,26 por cada 100 habitantes. Guantánamo con 4,13 fue la provincia con la tasa más elevada y Ciudad de La Habana con 2,13, la de más baja tasa. La mayor tasa de discapacidad se encontró en el grupo de 60 y más años y en el sexo masculino. La discapacidad intelectual ocupó el primer lugar en frecuencia, seguida de la discapacidad físico-motora. 
Conclusiones El número de personas con discapacidad en Cuba identificadas con los métodos empleados casi triplicó al universo inicial de discapacitados registrados, por lo que había una subestimación de la prevalencia real del problema. Las tasas de prevalencia de discapacidad y su distribución según características de las personas y provincias en Cuba siguen patrones similares a las descritas en otras publicaciones.

Palabras clave: Discapacidad, epidemiología, prevalencia, pesquisa activa, Cuba.

\section{ABSTRACT}

Introduction Disability is a complex topic, with great social and economic impact and inaccurate estimates.

Objectives Identify and characterize every disabled person in Cuba.

Methods Cross-sectional descriptive epidemiological research study carried out from 2001 to 2003 . The study covered the whole Cuban population, particularly the disabled persons. The community-wide active screening detected registered and non-registered persons with some disability, thanks to the support of local governments and social organizations. Home visits paid to disabled persons allowed combining interviews and clinical exams to facilitate the process of classification and registration of these people. The coverage rate of the study was estimated at $99,9 \%$ of the total number of disabled persons throughout the country.

Results The study analyzed 366864 people with disabilities, which led to a prevalence rate of 3,26 per 100 inhabitants. Guantanamo province reached 4,13, being the province with the highest rate whereas Ciudad de La Habana exhibited the lowest rate $(2,13)$. The highest rate of disability was found in the 60 and over years-old group and in males. Intellectual disability was prevailing in terms of frequency, followed by physical-motor disability.

Conclusions The number of individuals with some disability detected in Cuba was almost three times the initial universe of registered disabled, so the real prevalence of the problem had been underestimated. The disability prevalence rates and their distribution according to the characteristics of individuals and provinces in Cuba have patterns similar to those described in other publications.

Key words: Disability, epidemiology, prevalence, active screening, Cuba.

\section{INTRODUCCIÓN}

La discapacidad puede considerarse como un fenómeno universal, que se manifiesta en las personas en algún momento de su vida, sobre todo en la ancianidad, ${ }^{1}$ a pesar de lo cual todavía su definición no es obra terminada, coexisten diferentes maneras de enfocarla ${ }^{2,3} y$, con frecuencia, no es aceptada por quien la padece y sus allegados. Muchas veces permanece oculta por problemas de mayor magnitud, como la pobreza, que impiden o retardan el diseño y la aplicación de estrategias que permitan a esta gran masa de personas desarrollar sus capacidades y alcanzar la verdadera inclusión social, a la cual todos los seres humanos tienen derecho. 
La discapacidad es un tema complejo, de enorme repercusión social y económica, del que se dispone, quizás, tímidos e inseguros estimados. Los estudios

poblacionales no son frecuentes, son limitados o imprecisos; por ello, el trabajo en políticas públicas o programas relacionados con la discapacidad carece en muchos casos de la base científica y sociológica necesaria para la intervención correcta, efectiva y oportuna, que tropieza en no pocas ocasiones con la falta de voluntad de los gobernantes, sobre todo en los países del llamado "tercer mundo."

Entre las causas ambientales de la discapacidad pueden citarse: el envejecimiento de la población, las causas externas (accidentes de todo tipo, conflictos armados, inhalación y mala utilización de plaguicidas, uso y abuso de alcohol y drogas y la violencia social), además, la desnutrición, el abandono infantil y la marginación de grupos sociales como los pueblos indígenas; la pobreza extrema, el desplazamiento poblacional y los desastres causados por fenómenos naturales, todos los cuales, de una u otra manera, forman parte o están relacionados con los determinantes sociales de la salud. ${ }^{4,5}$

Este artículo, el primero que se realiza de una vasta investigación de carácter nacional, tiene como objetivo identificar y caracterizar a cada persona con discapacidad, por lo que pretende contribuir a la certidumbre necesaria sobre la real magnitud de este problema en el entorno latinoamericano y con ello mejorar el proceso de toma de decisiones en la atención a las personas con discapacidad.

\section{MÉTODOS}

Se realizó una investigación epidemiológica descriptiva, transversal en el período 2001-2003 en la República de Cuba. El estudio comprendió a toda la población cubana residente en el territorio nacional y dentro de ella incluyó a toda persona con discapacidad.

\section{Concepto de discapacidad}

Desde el punto de vista semántico, el término discapacidad está formado por el prefijo dis que significa separación y la palabra capacidad que significa inteligencia, talento y estado óptimo. Aunque el término es aún segregativo para catalogar a una persona, es mejor que otros, como minusválido.

A los efectos de la investigación, se consideró discapacidad a toda limitación grave que tenga una persona en la actualidad para realizar actividades, siempre que su duración total, es decir, el tiempo que la padece o espera padecer, sea superior a un año

Se incluyen las personas que han atenuado o eliminado su discapacidad con el uso de ayudas técnicas externas pero que tendrían dificultades importantes, si no dispusieran de dicha ayuda. ${ }^{6}$

\section{Criterios de inclusión ${ }^{6}$}

Se incluyeron en la investigación a las personas con discapacidad mayor, según los siguientes tipos de discapacidad recogidos en losl instrumentos utilizados:

\section{Discapacidad físico-motora mayor}

http://scielo.sld.cu 
- Parálisis de una extremidad superior o inferior.

- Hemiplejia, paraplejia o tetraplejia. Amputación de miembros superiores o inferiores.

- Trastornos en la coordinación de movimientos (involuntarios, temblores) y tono muscular (aumento o disminución).

- Trastornos graves del sistema nervioso (distrofias musculares, trastornos graves de la marcha).

- Alteraciones del sistema osteomioarticular o ausencias de miembros u otra región anatómica, deformidades graves de la columna vertebral. Enanismo, alteraciones desfigurativas severas.

Discapacidad visual

- Ceguera total: personas que no tienen percepción de luz en ninguno de los dos ojos.

- Débiles visuales (baja visión): personas con una grave deficiencia visual, no susceptible a tratamiento quirúrgico y que para ver de cerca requiere el uso de gafas con cristales o lupas con una potencia no menor a cuatro dioptrías.

Discapacidad auditiva

- Sordera: discapacidad para percibir cualquier sonido.

- Hipoacúsicos: personas con diferentes niveles de pérdida de la audición que requiere del uso de prótesis auditivas (audífonos).

\section{Discapacidad mental}

- Psicosis crónica: esquizofrenia, parafrenias y trastornos bipolares.

- Demencias: se refiere a cualquier tipo de enfermedad de Alzheimer, y a demencias vasculares y mixtas.

Discapacidad orgánica o visceral. Deficiencias de las funciones y estructura de los órganos.

- Insuficiencia renal crónica. Solo se contempló en la que existía criterio de diálisis.

Discapacidad mixta o múltiple. Cuando las personas están

afectadas por dos o más tipos de discapacidad mayor.

- La sordo-ceguera, discapacidad que se estudió como entidad propia, se define cuando la persona es ciega o débil visual asociado con una pérdida auditiva moderada o profunda demostrada.

\section{Criterios de exclusión ${ }^{6}$}

Se excluyeron las personas con: 
- Discapacidad menor.

- Retraso mental (en estudio independiente).

- Discapacidad temporal.

- Discapacidad somática o visceral, excepto la insuficiencia renal crónica.

El universo inicial de personas con discapacidad fue obtenido de diversas fuentes, especialmente de registros censales y de asociaciones de personas con discapacidad, también de personas dispensarizadas por discapacidad en la atención primaria de salud, así como de otras instituciones de salud y educación.

Los listados con la localización de las personas con discapacidad fueron entonces ajustados en visitas efectuadas a las comunidades con el auxilio de las autoridades locales y de las organizaciones de masas.

Tres rondas de estudios pilotos, permitieron adecuar y validar tres instrumentos utilizados para registrar y clasificar a las personas con discapacidad. El universo final de las personas con discapacidad casi triplicó al universo inicial. Se estimó una tasa de cobertura del estudio de un $99,9 \%$ del total de personas con discapacidad en el territorio nacional.

La actividad de campo se caracterizó en primer término por la pesquisa activa de las personas con discapacidad en la comunidad y en segundo lugar por la visita a sus hogares efectuada por un dúo integrado por un médico especialista en Medicina General Integral y un especialista en Psicopedagogía.

Se aplicó una combinación de entrevistas y exámenes clínicos como procedimiento de pesquisa y cuando fue indispensable y siempre con el consentimiento de la persona con discapacidad o su tutor, exámenes de laboratorio genético. Equipos multidisciplinarios de profesionales y técnicos, visitaron a la persona con discapacidad en su hogar, todas las veces que fue necesario hasta completar su atención.

Los datos registrados en los instrumentos eran captados diariamente, usando para ello una aplicación informática determinada. Se desarrollaron procesos de garantía de la calidad de los datos, tanto en el trabajo de campo por acciones sistemáticas de supervisión, como por rutinas de validación en la carga y procesamiento de los datos. Se construyeron distribuciones de frecuencias de variables de personas y lugar. Posteriormente se calcularon tasas de prevalencia usando como denominador la población media del período 2001-20003 para Cuba y sus territorios.

Al concluir el trabajo de campo, los datos eran rápidamente procesados y una información detallada de los resultados era comunicada a la comunidad y a sus líderes formales e informales.

La investigación permitió identificar además problemas y restricciones que afectaban las condiciones de vida de las personas con discapacidad, pero su solución y los diferentes procedimientos utilizados será motivo de próximas publicaciones.

\section{RESULTADOS}

http://scielo.sld.cu 
El total de personas con discapacidad en Cuba, ascendió a 366864 lo que se tradujo en una tasa de prevalencia en el país de 3,26 por cada 100 habitantes.

Su distribución por provincia se relaciona a continuación: Guantánamo fue la provincia con la tasa más elevada 4,13 por cada 100 habitantes; le siguen en orden descendente: Cienfuegos con 3,84 por 100 habitantes; Santiago de Cuba con 3,79 por cada 100 habitantes; Camagüey con 3,73 por cada 100 habitantes; Pinar del Río con 3,67 por cada 100 habitantes; Villa Clara y Ciego de Ávila con 3,64 por cada 100 habitantes, respectivamente; le sigue en frecuencias Las Tunas con 3,6 por cada 100 habitantes y Holguín con 3,59 por cada 100 habitantes, todas ellas por encima de la tasa media nacional.

Las provincias con el mayor número de personas con discapacidad, correspondieron a: Ciudad de La Habana (46 433), Santiago de Cuba (39 507), Holguín (37 181) y Villa Clara (30 419) (tabla 1).

Tabla 1. Prevalencia de personas con discapacidad según provincias. Cuba, 20012003

\begin{tabular}{|c|c|c|}
\hline Provincias & Total & Tasa x 100 hab. \\
\hline Pinar del Río & 27159 & 3,67 \\
\hline La Habana & 22021 & 3,09 \\
\hline Ciudad de La Habana & 46433 & 2,13 \\
\hline Matanzas & 21518 & 3,23 \\
\hline Villa Clara & 30419 & 3,64 \\
\hline Cienfuegos & 15299 & 3,84 \\
\hline Sancti Spíritus & 13901 & 3,00 \\
\hline Ciego de Ávila & 15035 & 3,64 \\
\hline Camagüey & 29537 & 3,73 \\
\hline Las Tunas & 19194 & 3,60 \\
\hline Holguín & 37181 & 3,59 \\
\hline Granma & 25777 & 3,08 \\
\hline Santiago de Cuba & 39507 & 3,79 \\
\hline
\end{tabular}




\begin{tabular}{|l|c|c|}
\hline Guantánamo & 21306 & 4,13 \\
\hline Isla de la Juventud & 2577 & 2,93 \\
\hline Total & 366864 & 3,26 \\
\hline
\end{tabular}

Fuente: Estudio psicosocial y clínico genético de las personas con discapacidad.

Al considerar la prevalencia por grupos de edades (tabla 2) la mayor frecuencia fue observada en el grupo de 60 y más años, con una tasa de 7,8 por cada 100 habitantes, también fue perceptible un incremento con la edad en los primeros tres grupos para caer ligeramente entre los 29 y 49 años y ascender nuevamente en el de 60 y más años.

Tabla 2. Prevalencia de personas con discapacidad según grupos de edades. Cuba, 2001-2003

\begin{tabular}{|l|c|c|c|}
\hline $\begin{array}{l}\text { Grupo de edad } \\
\text { (años) }\end{array}$ & Población & No. & Tasa x 100 hab. \\
\hline $0-4$ & 743517 & 4399 & 0,6 \\
\hline $5-14$ & 1634368 & 36752 & 2,3 \\
\hline $15-19$ & 775991 & 22957 & 3,0 \\
\hline $20-29$ & 1715449 & 41672 & 2,4 \\
\hline $30-39$ & 2169619 & 53365 & 2,0 \\
\hline $40-59$ & 2612250 & 81100 & 2,1 \\
\hline 60 y más & 1606892 & 126619 & 7,8 \\
\hline Total & 11258086 & 366864 & 3,26 \\
\hline
\end{tabular}

Fuente: Estudio psicosocial y clínico genético de las personas con discapacidad.

La prevalencia de discapacidad en el sexo masculino con tasa de 3,57 por cada 100 habitantes fue mayor que en el sexo femenino, el que mostró una tasa de 2,99 por cada 100 habitantes (tabla 3). 
Tabla 3. Prevalencia de personas con discapacidad según sexo. Cuba, 2001-2003

\begin{tabular}{|l|c|c|c|}
\hline Sexo & No. & $\%$ & Tasa x 100 hab. \\
\hline Femenino & 167239 & 45,58 & 2,99 \\
\hline Masculino & 199625 & 54,42 & 3,57 \\
\hline Total & 366864 & 100,0 & 3,26 \\
\hline
\end{tabular}

Fuente: Estudio psicosocial y clínico genético de las personas con discapacidad.

Al caracterizar las personas con discapacidad según tipo (tabla 4), la discapacidad intelectual se presentó en Cuba como la discapacidad más frecuente, con una tasa de prevalencia de 1,25 por cada 100 habitantes (140 489 personas) y representó el $38,29 \%$ del total de personas con discapacidad, seguido de la discapacidad físico motora con tasa de prevalencia de 0,82 por 100 habitantes (92 506 personas) el $25,22 \%$ del total de personas con discapacidad. Continuaron en orden de frecuencia, la discapacidad de tipo visual (tasa 0,41 por 100 habitantes y $12,7 \%$ del total); la mental (tasa 0,33 por 100 habitantes y 10,0\% del total); la múltiple (tasa 0,22 por 100 habitantes y 6,8 \% del total); la auditiva (tasa 0,21 por 100 habitantes y $6,4 \%$ del total) y finalmente la insuficiencia renal crónica con requerimientos dialíticos (tasa 0,02 por 100 habitantes y 0,5\% del total). 
Tabla 4. Prevalencia de personas con discapacidad según tipo. Cuba, 2001-2003

\begin{tabular}{|l|c|c|c|}
\hline Tipo de discapacidad & No. & Tasa x 100 hab. \\
\hline Intelectual & 140489 & 38,3 & 1,25 \\
\hline Físico motora & 92506 & 25,2 & 0,82 \\
\hline Visual & 46455 & 12,7 & 0,41 \\
\hline Mental & 36869 & 10,0 & 0,33 \\
\hline Múltiple & 25094 & 6,8 & 0,22 \\
\hline Auditiva & 23620 & 6,4 & 0,21 \\
\hline Visceral (IRC) & & & \\
\hline & 1831 & 0,5 & 0,02 \\
\hline Total & 366864 & 100,0 & \\
\hline & & & \\
\hline
\end{tabular}

* Insuficiencia renal crónica con requerimientos dialíticos.

Fuente: Estudio psicosocial y clínico genético de las personas con discapacidad.

\section{DISCUSIÓN}

Los estudios poblacionales relacionados con la discapacidad no son frecuentes. El hecho de que la discapacidad puede ser definida desde diferentes perspectivas, hace que los estudios publicados ofrezcan cifras de frecuencia que pueden resultar muy diferentes, aun cuando las poblaciones en las que se hayan realizado puedan tener muchos elementos comunes.

La mayoría de los reportes sobre magnitud de la discapacidad se realizan a partir de estudios censales, encuestas de población o sobre muestras de población con mayor o menor grado de representatividad; también se publican datos basados en "demanda satisfecha", es decir, información obtenida en servicios de salud que documentan la frecuencia relativa de solicitud de atención de personas con tal o cual tipo de discapacidad o de enfermedad o de instituciones a las cuales se solicitan prestaciones. ${ }^{9}$

Se estima unos 650 millones de personas con discapacidad en el mundo. De ellos siete de cada diez viven en países pobres, es decir más de 450 millones con personas con discapacidad, lo que la coloca como problema mundial. ${ }^{10}$

En Cuba, las tasas de prevalencia se movieron en las diferentes provincias entre 2,13 y 4,13 por 100 habitantes. Esta variabilidad podría ser explicada, entre otros factores, por el grado de desarrollo socioeconómico desigual que exhiben los 
diferentes territorios y en algunos casos por la marcada presencia de algunos otros determinantes de la discapacidad.

Las tasas de prevalencia de discapacidad en Cuba resultan iguales o discretamente inferiores a las cifras reportadas internacionalmente. ${ }^{11}$ En Centroamérica, Collado ${ }^{7}$ informa para el Salvador 1,5 \% (Encuesta 2004), para Guatemala 3,7 \% (Encuesta 2005), para Honduras 2,7\% (Encuesta 2002) para Nicaragua 10,3\% (Encuesta 2003) y para Panamá $11,3 \%$ (Encuesta 2005). Vásquez ${ }^{4}$ registra la prevalencia de discapacidad para otros países latinoamericanos como sigue: Argentina 6,8 \%, Bolivia 9,3\%, Brasil 6,0 \%, Chile 6,4 \%, Colombia 12,0 \%, Ecuador 13,2 \%, México $10,0 \%$, Perú $31,3 \%$, Uruguay $15,0 \%$ y Venezuela $10,00 \%$.

En Europa, Gispert ${ }^{12}$ notifica para España una prevalencia de 8,9 \%. Esta 